\title{
Urethtral Approach in Retroperitoneoscopic Radical Prostatectomy: A Novel Technique for Safe Prostate Dissection
}

\author{
Tetsuo Nozaki Akihiro Morii Yasuyoshi Fujiuchi Hideki Fuse \\ Department of Urology, Graduate School of Medicine and Pharmaceutical Sciences for Research, \\ University of Toyama, Sugitani, Toyama, Japan
}

\section{Key Words}

Laparoscopic radical prostatectomy • Urethral assistance • Traction $\cdot$ Prostate apex dissection

\begin{abstract}
Background and Objectives: Laparoscopic radical prostatectomy (LRP) remains technically challenging and is associated with a steep learning curve. Prostate apex dissection (PAD) is one the most challenging elements of LRP. This study describes a novel technique for PAD using a transurethral approach (urethral assistance). Methods: Through the transurethral route, Young's foreign body forceps is introduced under laparoscopic vision and both vas deferens are grasped for efficient traction of the prostate. The forceps is quickly clamped to the laparoscope holder. Urethral assistance facilitates more efficient dissection during dissection of the bladder neck, vas deferens, and seminal vesicles, transaction of the posterior prostatovesical junction, mobilization of the prostate off the rectum, and ligation of the dorsal venous complex. Results: In 10 patients with localized prostate cancer who underwent LRP using the urethral assistance technique, sufficient retraction of the prostate was maintained in the appropriate direction. No complications related to urethral assistance were observed. No internal or external instrument collisions occurred during PAD. Conclusion: Urethral assistance provides a simple, reliable, cost-effective approach for performing PAD during LRP.
\end{abstract}

Copyright $\odot 2013$ S. Karger AG, Basel

\section{KARGER}

Fax +4161306 1234

E-Mail karger@karger.ch

www.karger.com
(C) 2013 S. Karger AG, Basel

1015-9770/13/0072-0103\$38.00/0

Accessible online at:

www.karger.com/cur

\section{Introduction}

Recent reports have described laparoscopic radical prostatectomy (LRP) as reproducible, oncologically equivalent to other methods, and beneficial to patient outcomes [1, 2]. However, LRP remains technically challenging and is associated with a steep learning curve, requiring extensive training because of its technical difficulty [3-7]. During the learning period, prostate apex dissection (PAD) is one the most challenging elements of LRP because of the limited field of view in the small pelvis area. Maintenance of optimal exposure is critical to the efficient and safe completion of PAD. This study describes a novel technique to maintain optimal exposure by a transurethral approach (urethral assistance; UA) using the laparoscope holder to facilitate traction of the prostate during PAD.

\section{Surgical Technique}

Patients are positioned in the horizontal dorsal decubitus position with Y-shaped abduction of lower limbs on the table and arms placed at the sides. A retroperitoneal approach is followed using five trocar ports. Exeresis of preprostatic fat using monopolar cautery is performed for correct identification of prostate and bladder. Lymphadenectomy involving the obturator nerve is performed. Thereafter, the junction of the endopelvic fascia is bluntly divided. After the boundary between the prostate and

Tetsuo Nozaki

Department of Urology

Graduate School of Medicine and Pharmaceutical Sciences for Research

University of Toyama

2630 Sugitani

JP- 930-0194 Toyama (Japan)

E-Mail nozaki0921@yahoo.co.jp 

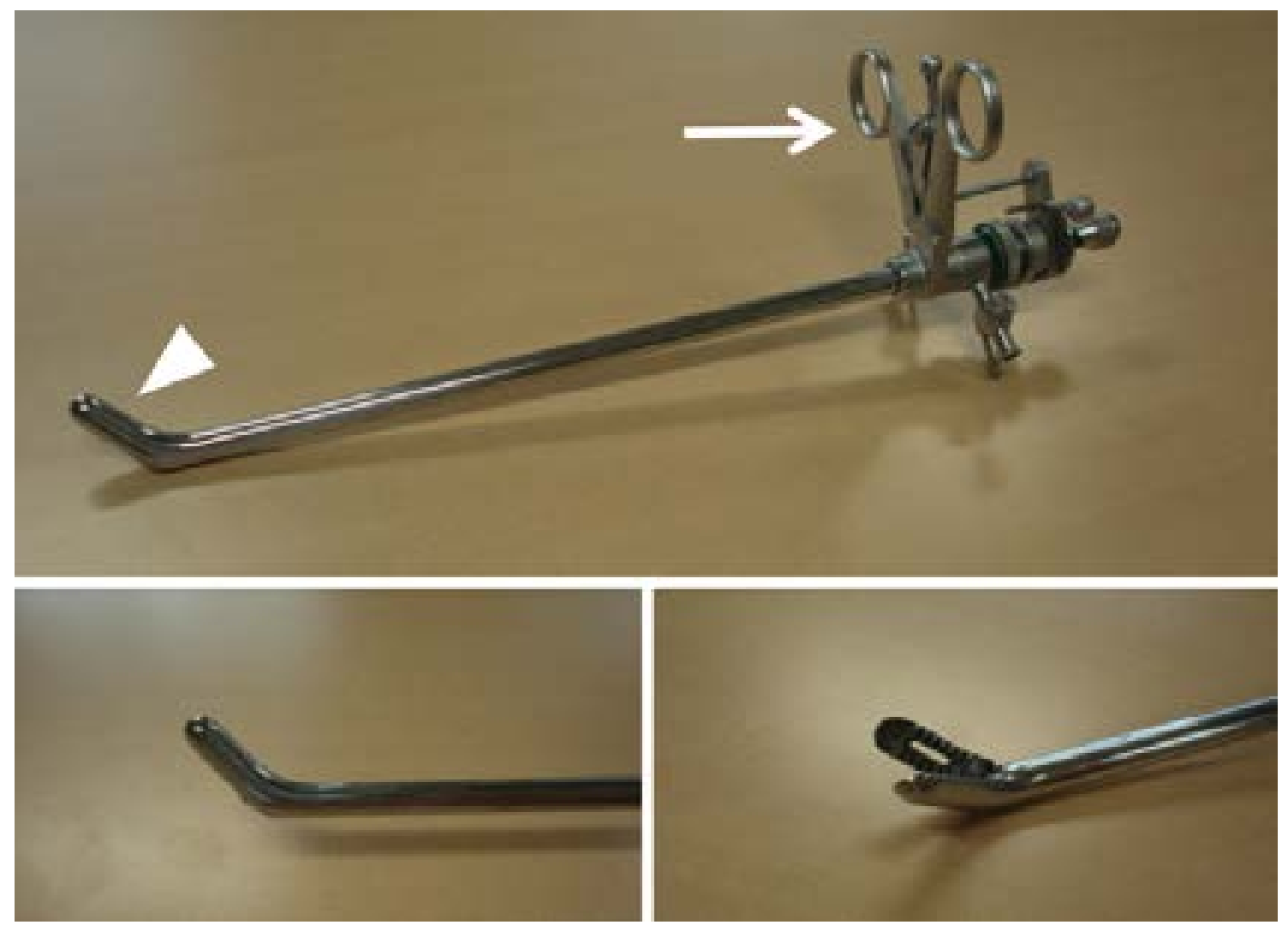

Fig. 1. Young's foreign body forceps. Angled jaws at the tip (arrowhead) and ratcheted handles at the end (arrow) of Young's foreign body forceps.

bladder neck has been confirmed, the muscle layer of the bladder is detached from the prostate as if peeling the bladder from the basal prostate, preserving the bladder neck as much as possible. Dissection is performed from the anterior neck incision until the Foley catheter becomes visible. At this point in the procedure, a laparoscope holder (Karl Storz GmBH and Co., Tuttlingen, Germany) is quickly clamped to the table on the left side through a base rod over a sterile drape. The laparoscope holder consists of 4 components: a table clamp, a base rod, an articulating arm, and a precision clamp. The precision clamp, which is located at the tip of the holding system, accommodates instruments of various sizes. The articulating arm has three joints and is operated manually. Young's foreign body forceps (fig. 1) is transurethrally introduced under laparoscopic vision. The tip of the forceps is introduced at the window of the anterior opening of bladder neck. The prostate is then retracted towards the abdominal wall to optimize exposure. Once it is in the correct position, the set screw on the handle is tightened, thereby precluding any inadvertent release of traction on the prostate (fig. 2). The bladder neck incision is extended horizontally, and the posterior aspect of the bladder neck is identified and dissected at the prostatovesical junction, starting from the midline and extending laterally to completely detach the bladder neck of the prostate base. After dissection of the prostate base, applying traction on the prostate using Young's forceps helps in easier identification of the vas deferens (VD). Both VD are identified and cut, and a long stump is maintained for retraction. Young's forceps are used to grasp both VD as low as possible for efficient traction of the prostate (fig. 3 ). The vas is pulled into the most anterior position possible, thus exposing the seminal vesicles located lateral to VD on either side. After complete release of seminal vesicles, the Denonvilliers' fascia is easily identified. UA provides sufficient stretch to maintain traction on the prostate, thereby elevating it away from the rectum. The 


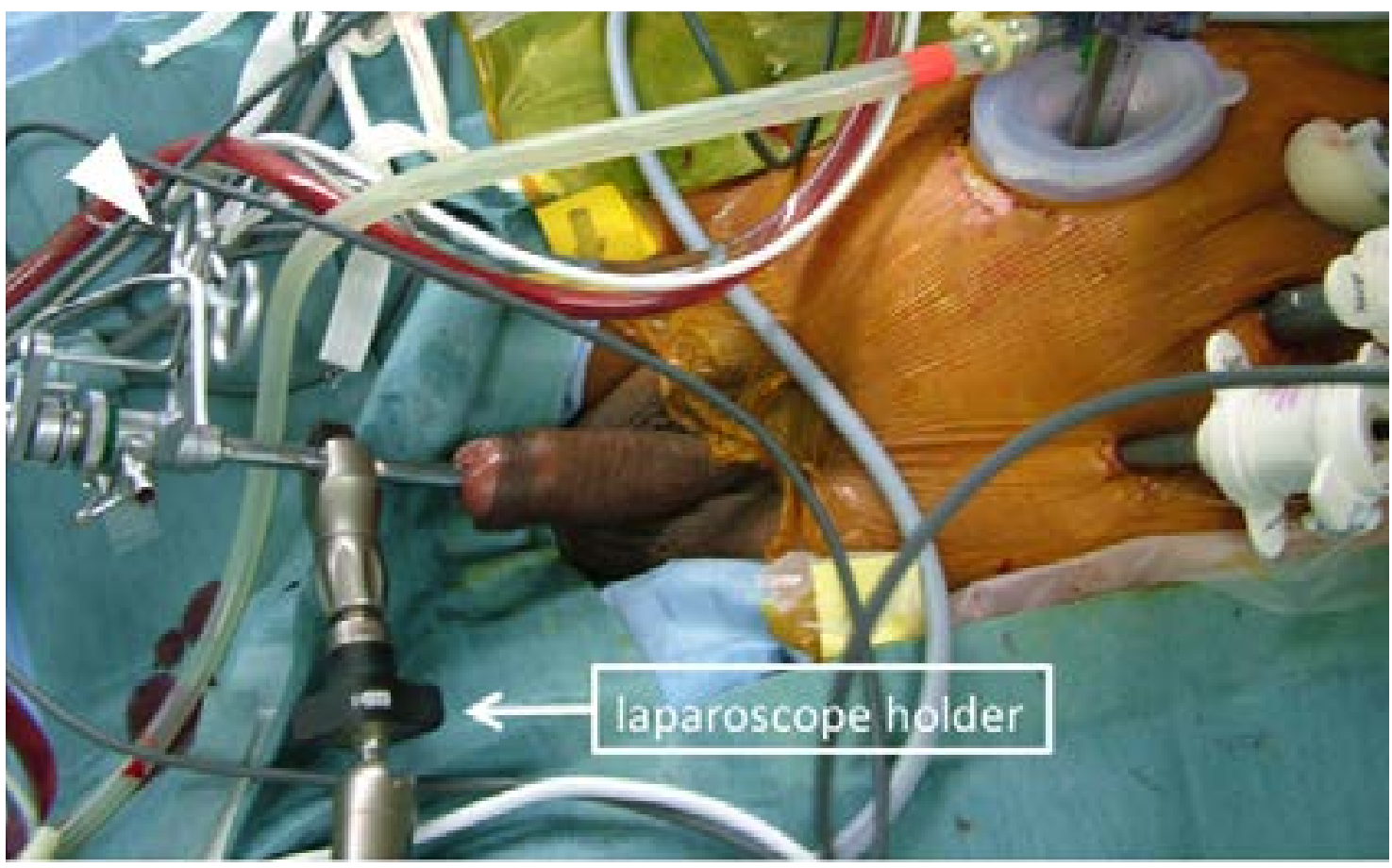

Fig. 2. Once in the correct position, the handle is tightened and Young's foreign body forceps (arrowhead) was fixed by the laparoscope holder.

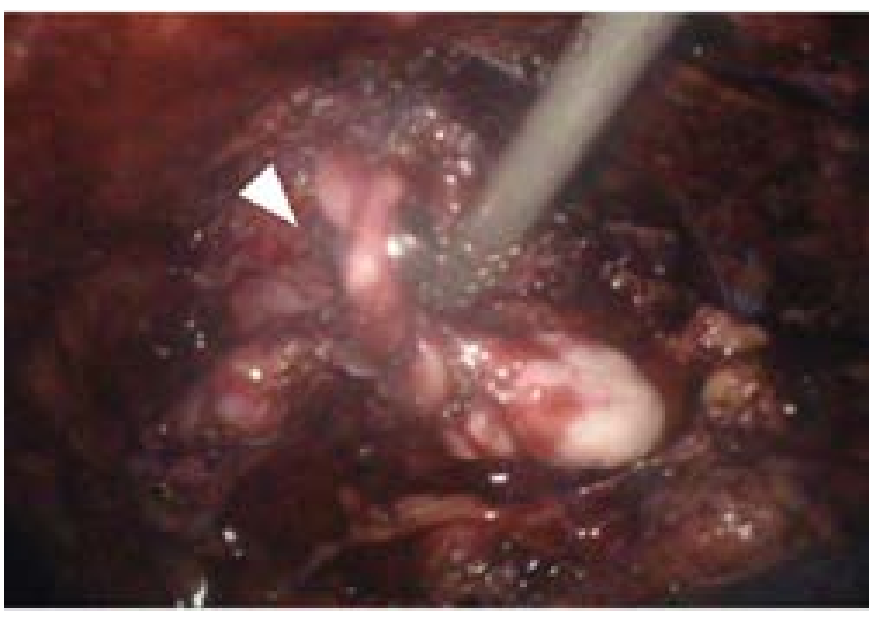

Fig. 3. Young's foreign body forceps (arrowhead) grasping both $\mathrm{VD}$ as low as possible for efficient traction of the prostate.

Denonvilliers' fascia is then cut horizontally. The rectum is bluntly dissected from the dorsal surface of the prostate, separating the rectal side of the lateral pedicle from the prostate. During dissection of the lateral pedicle, UA allows changes in the direction of traction on the prostate to the left or right of the pelvis to facilitate dissection of the lateral wing at these points in the prostate apex (fig. 4a). Sufficient tension enables tissues to be separated by retraction, making the PAD procedure easier to manage (fig. 4b). The rectum must only be pulled inferiorly to prevent rectal injury. If required, minor repositioning of the device can easily be performed. The laparoscope holder also secures the retractor in the desired position. Dissection is deliberately extended distally to release the apex from deeper tissues.

Next, the approach is changed to the apical-urethral junction. Young's forceps is gently maneuvered horizontally in the cephalad direction out of the pelvis. The laparoscope holder maintains an adequate view of the prostate apex. The prostate apex is stretched (fig. 5), "presented" to the surgeon, ready for ligation of the dorsal venous complex (DVC). The puboprostatic ligaments are cut using laparoscopic scissors. The prostate is pulled in the cephalad direction out of the pelvis, providing countertraction. The entire DVC is then double-ligated with a 17-cm 2-0 Vicryl suture (MH needle; Ethicon, Norderstedt, Germany) as distantly as possible. After cutting DVC, the anterior urethra is meticulously peeled 

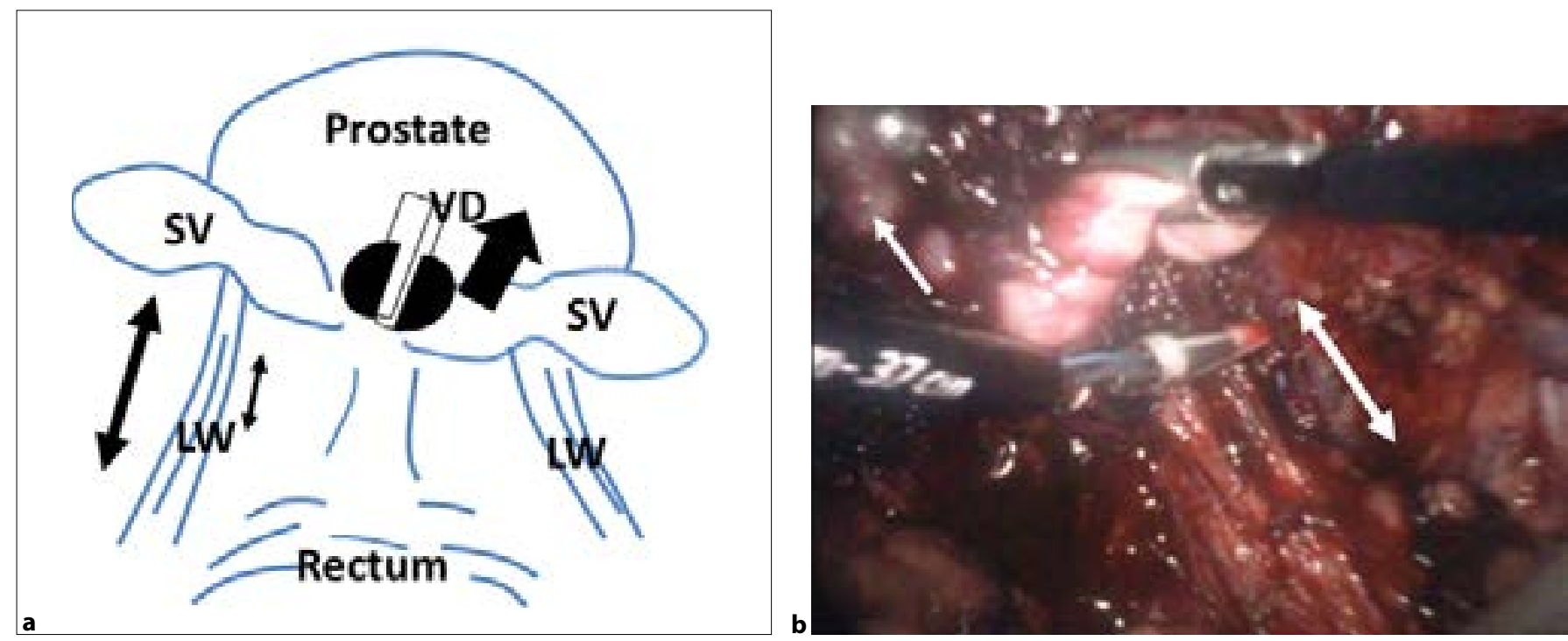

Fig. 4. a The direction of traction on the prostate can be easily, quickly, and precisely changed depending on the site of dissection of the lateral wing (LW). b PAD of the right side. Sufficient tension facilitates dissection of the lateral wing $(\mathrm{LW})$.

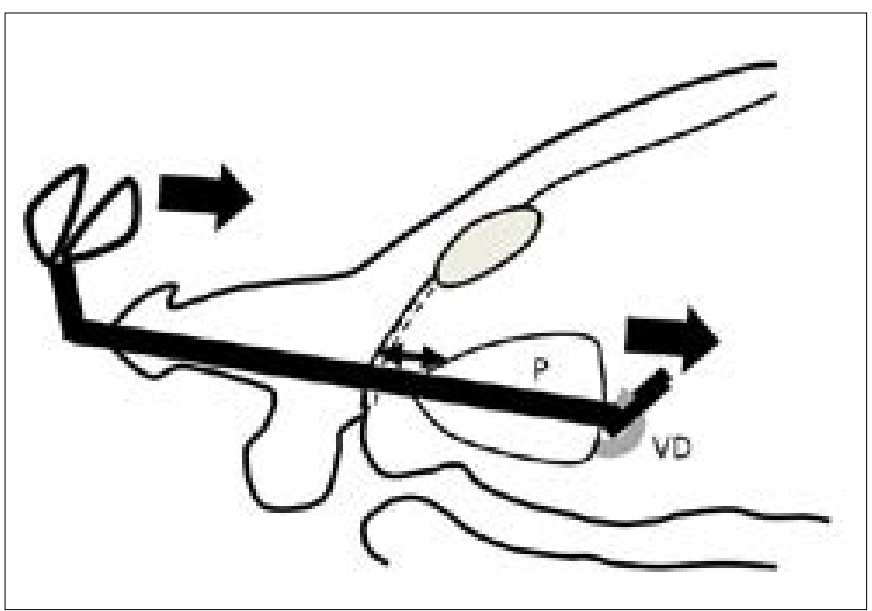

Fig. 5. Young's foreign body forceps is gently maneuvered horizontally in the cephalad direction out of the pelvis to maintain an adequate view of the prostate apex. After traction of the prostate, the prostate apex is adequately stretched.

from the surrounding tissue. The resection line is set as proximally as possible, providing countertraction to the urethra, which is extended to its maximum possible length to ensure that no prostate tissue remains. The urethra is incised circumferentially, after which the prostate is freed. Urethrovesical anastomosis is performed using continuous sutures.

\section{Results}

Ten patients with localized prostate cancer underwent LRP using the UA technique in Toyama University Hospital from November 2011 to November 2012. In all cases, the prostate was adequately retracted using the method described above, and the apex of the prostate was fully exposed. The laparoscope holder maintained retraction of the prostate in the appropriate direction in all cases. No complications related to the use of the laparoscope holder were observed. Less than 5 minutes were required to prepare and position the device. Moreover, no internal or external instrument collisions occurred during PAD.

\section{Discussion}

Adequate exposure is critical for the efficient and safe execution of all surgical procedures. Exposure is even 
more important in laparoscopic surgery, because visual information must substitute for the sense of touch. In conventional LRP, the surgeon was originally equipped with five ports, two of which were used as working ports, the third for the endoscope, and two additional ports were then handled by one trained assistant. Especially during PAD, the conventional LRP approach greatly relied on assistants for execution of several key steps, including dissection, irrigation of the operative field, suction of oozing blood for better exposure, and tissue traction. Optimal exposure and tissue traction are difficult to maintain because tactile feedback is not possible during laparoscopic PAD. Appropriate countertraction with a laparoscopic retractor from numerous angles was sometimes technically difficult and problematic. Although a skillful assistant can successfully retract the prostate, these maneuvers are occasionally beyond the capacity of surgical assistants. Therefore, the novel technique presented here was developed.

This novel technique, UA improves exposure and tissue traction in the narrow retroperitoneal space. Because the apex is the narrowest part of the prostate, in conventional LRP, the narrow operative field is crowded by various surgical instruments. Using the urethral approach, internal and external collisions or other difficulties in manipulating surgical instruments, problems typical of PAD procedures, are avoided. UA maintains adequate isometric force for optimal exposure for a prolonged period. In conventional laparoscopic organ traction, potential disadvantages include the difficulty of maintaining isometric force at a constant level and maintaining optimal exposure as the steps of the surgical procedure increase in complexity. As a result of muscle fatigue in the assistant surgeon, the retractors may accidentally move or excessive force may inadvertently be applied when the prostate is retracted. Moreover, because of the difficulties associated with a limited range of motion of the laparoscopic instruments in the small pelvis area, insufficient traction may occur during conventional LRP.

Young's foreign body forceps is the grasping type forceps with angled jaws at the tip. This instrument was originally designed for transurethral cystolitholapaxy to treat adults with bladder stones. In our procedure, Young's foreign body forceps was used for grasping VD and simultaneously retracting the prostate. The angled jaws at the tip are designed and fitted for transurethral insertion. Once the base of the VD is grasped, because of the shape of the angled jaws, the prostate is spontaneously elevated towards the abdominal wall. Therefore, only minor repositioning is necessary to provide sufficient retraction to optimize exposure. Once in the correct position, the handle is tightened and fixed by the laparoscope holder, thereby preventing instrument migration caused by muscle fatigue. In UA, traction of the prostate is performed at the base of VD to provide sufficient force and tension so tissues can be separated by retraction. The direction of traction on the prostate from the Young's foreign body forceps can be easily changed as necessary depending on the site of dissection of the lateral wing. These features of LRP using UA alleviate many of the difficulties of the PAD procedure. Moreover, this technique avoids excessive traction on the prostate itself, minimizing fascial tearing during oncologic procedures.

In addition, the additional urethral working reduces dependence and reliance on assistants. One of the assistant's hands is free to assist as needed; moreover, instead of maintenance of the surgical field, the assistant can concentrate on suction/irrigation and, on rare occasions, additional retraction using the other hand. LRP by the transurethral route using Young's forceps in the UA technique gives the surgeon independent assistance during PAD. UA allows the assistant to play a smaller and less stressful role while constantly maintaining the proper retractor position. Depending on the institution, prostate retraction could even be performed by the fourth scrubbed surgical assistant with a urethral bougie. However, participation of four assistants may be impractical, especially in community hospitals and private institutions. Introduction of positioning systems for laparoscopic procedures may be a more suitable option for community urologists with limited personnel resources. Moreover, since the main laparoscopic view in most procedures is focused on the surgical site and not the retracted prostate, the likelihood of inappropriate traction may increase. Human assistance is costly and may not provide complete satisfaction to the surgeon. The UA technique described here may thus alleviate some of the difficulties of performing LRP inherent in smaller institutions.

To the best of our knowledge, this useful technique has never been reported in open radical prostatectomy, LRP or robotic LRP. The disadvantage of using Young's forceps is that a 26-F instrument is situated in the urethra for a prolonged period, which may cause further urethral trauma and predispose formation of urethral stricture. However, these complications were not observed in the cases reported here. Although no comparative outcome data with and without the technique is available, we feel that the LRP procedure using the UA technique offers sufficient advantages in visualization of the prostate apex, recommending it as standard practice. 


\section{Conclusions}

This report presents initial clinical experiences with the UA technique, which provides a simple, reliable, cost-effective approach to performing PAD. The UA system provides considerable freedom to perform retraction and improved tissue exposure during key steps in PAD. The addition of UA simplifies the assistant's role during surgery. UA facilitates dissection of the bladder neck, $\mathrm{VD}$, and seminal vesicles, transaction of the posterior prostatovesical junction, mobilization of the prostate off the rectum, ligation of DVC, and dissection of the vascular pedicles. UA gives the operating surgeon an added degree of independence, thus allowing more efficient dissection through the primary working ports.

\section{References}

$>1$ Gill IS, Zippe CD: Laparoscopic radical prostatectomy: technique. Urol Clin North Am 2001;28:423-436.

$>2$ Rassweiler J, Stolzenburg J, Sulser T, Deger S, Zumbé J, Hofmockel G, John H, Janetschek G, Fehr JL, Hatzinger M, Probst M, Rothenberger KH, Poulakis V, Truss M, Popken G, Westphal J, Alles U, Fornara P: Laparoscopic radical prostatectomy: experience of the German Laparoscopic Working Group. Eur Urol 2006;49:113-119.
Ahlering TE: Robotic versus laparoscopic radical prostatectomy. Nat Clin Pract Urol 2004;1:58-59.

4 Tooher R, Swindle P, Woo H, Miller J, Maddern G: Laparoscopic radical prostatectomy for localized prostate cancer: a systematic review of comparative studies. J Urol 2006; 175:2011-2017.

$>5$ Kavoussi LR: Laparoscopic radical prostatectomy: irrational exuberance? Urology 2001;58:503-505.
6 Guillonneau B, Rozet F, Cathelineau X, Lay F, Barret E, Doublet JD, Baumert H, Vallancien G: Perioperative complications of laparoscopic radical prostatectomy: the Montsouris 3-year experience. J Urol 2002;167: 51-56.

7 Trabulsi EJ, Guillonneau B: Laparoscopic radical prostatectomy. J Urol 2005;173:10721079. 